\title{
Efectos De La Fertilización Nitrogenada Y Fosfatada Sobre Poblaciones De Micorrizas Asociadas Al Cultivo De Cacao
}

\author{
Ing. Agr. Wilson Latacela Coello \\ Ing. Agr. Eduardo Colina Navarrete \\ Ing. Ind. Carlos Castro Arteaga \\ Ing. Agr. Danilo Santana Aragone \\ Ing. Agr. Joffre León Paredes \\ Ing. Agr. Guillermo García Vásquez \\ Ing. Agr. Miguel Goyes Cabezas \\ Ing. Agr. Maribel Vera Suarez.
}

Investigadores de la Facultad de Ciencias Agropecuarias Universidad Técnica de Babahoyo, Ecuador

doi: 10.19044/esj.2017.v13n6p464 ～URL:http://dx.doi.org/10.19044/esj.2017.v13n6p464

\begin{abstract}
Cocoa is one of the most significant assets in the ecuadorian agricultural structure, which also provides a good source of income for thousands of farmworker families. However, fertilization research works in cocoa cultivation have been relatively scarce. Decades of global research have shown that mycorrhizal fungi determine the fertilization and conservation of agrosystems. Fertilization is considered to be among the cultural and agricultural practices affecting mycorrhizas, which diminishes or prevents the fungal colonization. The objectives of research works were the following: the characterization of mycorrhiza species associated with cocoa, to determine the effects of nitrogen and phosphate fertilization in plant population and to identify the critical dose affecting the fungus development. A factorial design was used $(\mathrm{AxBxC})$, with two (2) application systems, four (4) doses of nitrogen fertilizer, and two (2) doses of phosphate fertilizer with three repetitions. The turkey's test was used to evaluate means with confidence interval of $95 \%$. The evaluated variables were: diameter and length of cocoa pods, number of cocoa pods per tree, index of cocoa beans and almond yield per hectare, as well as, mycorrhizal spore counting, percentage of mycorrhizal fungi colonization, visual density of endophyte, and characterization of mycorrhiza species. The results concluded that the application of $100 \mathrm{Kg} / \mathrm{ha}$ $\mathrm{N}+70 \mathrm{~kg} / \mathrm{ha} \mathrm{P}$ in the system below the soil surface, achieved yield increments
\end{abstract}


of $68 \%$ over the absolute witness. The same dose and application method, increased the number of spores per gram of soil, mycelium colonization and density of mycorrhizal endophyte in roots.

Keywords: Sustainable Agriculture, Cocoa, Mycorrhizas, Fertilization, Nitrogen.

\section{Resumen}

El cacao es uno de los rubros de mayor relevancia en la estructura agrícola de Ecuador y fuente de ingreso para miles de familias campesinas. Los trabajos de investigación sobre fertilización en el cultivo del cacao, han sido relativamente escasos. A nivel mundial décadas de investigación muestran que las micorrizas determinan la fertilización y conservación de los agrosistemas. Entre las prácticas culturales agrícolas que afectan a las micorrizas está la fertilización, la cual merma la colonización del hongo. Los objetivos del trabajo fueron: caracterizar las especies de micorrizas asociadas al cacao, determinar el efecto de la fertilización nitrogenada y fosfatada sobre las poblaciones e identificar la dosis crítica que afecte el desarrollo del hongo. Se empleó un diseño factorial $\mathrm{AxBxC}$, con dos sistemas de aplicación, cuatro dosis de fertilizante nitrogenado y dos de fertilizante fosfatado, en tres repeticiones. Para la evaluación de medias se utilizó la prueba de Tukey al 95\% de confianza. Las variables evaluadas fueron: diámetro de mazorca, longitud de mazorca, número de mazorcas por árbol, índice de semillas y rendimiento por hectárea de almendras, conteo de esporas, porcentaje de colonización de micorrizas, densidad visual del endófito, caracterización de especies micorrízicas. Los resultados establecieron que la aplicación de 100 $\mathrm{kg} / \mathrm{ha} \mathrm{N}+70 \mathrm{~kg} / \mathrm{ha} \mathrm{P}$ en el sistema bajo superficie, logró incrementos de rendimiento del $68 \%$ más sobre el testigo absoluto. La misma dosis y método de aplicación, aumentó el número de esporas por gramo de suelo, colonización de micelio y densidad de endófito micorrízico en las raíces.

Palabras claves: Agricultura Sostenible, Cacao, Micorrizas, Fertilización, Nitrógeno.

\section{Introducción}

El cacao es uno de los rubros de mayor relevancia en la estructura agrícola productiva de Ecuador y fuente de ingreso para miles de familias campesinas. Se cultiva en Los Ríos (Vinces, Babahoyo, Palenque Baba, Pueblo Viejo, Catarama y Ventanas), Guayas (Naranjal, Balao y Tenguel) y El Oro (Machala y Santa Rosa), en mayor cantidad. El promedio de área sembrada por agricultor es de 3 hectáreas, los rendimientos anuales fluctúan entre 300 a $500 \mathrm{~kg} / \mathrm{ha}$ de cacao seco (ANECACAO, 2009). 
En el caso de la fertilización, hay muy pocos estudios de investigación, por lo cual se encuentra muy poca información sobre las dosis y épocas de aplicación de fertilizantes de acuerdo con la edad y grado de sombrío de las plantaciones en las diferentes zonas productoras del país. La creencia del cacao es poco productivo, desalienta los proyectos de desarrollo vinculados al cultivo. Además una huerta sin tecnología agrícola contribuye a que el problema se mantenga con productividades de 0.25 toneladas métricas por hectárea. La nueva investigación apunta a lograr mejorar prácticas culturales (principalmente fertilización) para mejorar el sistema de cultivo (INIAP, 2010).

Una situación problemática bastante generalizada, es la mala aplicación de fertilizantes en el suelo con el fin de aumentar el rendimiento de las cosechas, en esos instantes los fertilizantes pierden su acción beneficiosa y pasan a ser contaminantes del suelo.

Los hongos micorrízicos son especies con la capacidad de colonizar el exterior o interior de las raíces de absorción de plantas, para obtener compuestos orgánicos esenciales. Las micorrizas son capaces de absorber, acumular y transferir los principales macro y micro nutrientes, y el agua a la planta más rápidamente que las raíces sin micorrizas. Décadas de investigación muestran que las micorrizas incrementan la tolerancia de las plantas a la sequía, compactación, altas temperaturas del suelo, metales pesados, salinidad, toxinas orgánicas e inorgánicas y extremos de $\mathrm{pH}$ del suelo (Sieverding, 2007).

Las micorrizas poseen características tanto químicas como físicas y a su vez biológicas que determinan la fertilización y conservación de los agro sistemas, la actividad de estos microorganismos influyen en la cinética que se lleva a cabo en los suelos cultivados como no cultivado tales como: la mineralización e inmovilización de nutrientes y su participación activa en el ciclo de los nutrientes del suelo (Modjo et al., 2006).

Los microorganismos benéficos están llegando a causar un juego fundamental en el suelo, entre estos se encuentran los microorganismos como: micorrizas arbusculares (MA), los microorganismos fijadores de nitrógeno y las rizobacterias causantes del crecimiento vegetal. Muchos proyectos han mostrado como influyen estos en el desarrollo, crecimiento y resistencia a enfermedades de la planta, sin embargo para sostener los sistemas de producción agrícola en el Ecuador es necesario conocer que los integran y pueden llevar a una alta productividad ya que esto se debe a la fertilidad de los suelos (Bastidas et al., 2005).

Con la utilización de las micorrizas como biofertilizantes no se suprime la aplicación de fertilizantes, sino que fertilizar se vuelve más eficiente y puede disminuirse la dosis a aplicar desde comúnmente 50-80 \% y en ocasiones hasta un $100 \%$. Se plantea que de las cantidades de fertilizantes aplicadas, sólo se 
aprovecha un $20 \%$, y el resto normalmente se filtra o se lixivia sin remedio; con la aplicación de los hongos micorrízicos, se recupera un porcentaje mucho mayor; ya que las hifas del micelio pueden captar más nutrimentos hasta 40 veces mayor (Pérez, 2000).

Varios factores afectan las micorrizas arbusculares, entre ellos la fertilización y labranza, causan mermas en la colonización del hongo. Toda esta situación provoca condiciones desfavorables para nutrición de los cultivos, por lo que se hace necesaria la búsqueda de nuevas alternativas de fertilización, teniendo en cuenta que entre los principales elementos en la nutrición de los cultivos se encuentran el Nitrógeno y el Fósforo, muy importantes para el desarrollo y crecimiento de los cultivos agrícolas.

Es importante indicar que por la dependencia micorrizica del cacao (Theobroma cacao L.), la práctica agronómica de la fertilización, especialmente nitrogenada y fosfatada, debe ser evaluada en su integridad, considerando no sólo el incremento en el rendimiento del cultivo y en la disponibilidad de nutrientes en el suelo, sino también en la composición y comportamiento de los microorganismos, con el objetivo de no interrumpir el ciclo de trasformación de nutrientes, ni tampoco incentivar la dependencia de insumos externos en el sistema (López et al., 2000).

El presente trabajo de investigación contribuyo en gran medida en la reducción del costo de los programas de fertilización, aprovechando las micorrizas de suelos y su efecto sobre la asimilación de nutrientes. Además permitió una integración con el sector productivo de la provincia en especial con el pequeño productor, favoreciendo el conocimiento sobre los recursos con los que cuenta la finca.

\section{Materiales y métodos}

La presente investigación se realizó durante los meses de enero a diciembre del año 2015, en la finca "El Recuerdo", perteneciente a la parroquia Pimocha, localizada al Noroeste del cantón Babahoyo. La misma que se estableció en una plantación cacaotera de esos predios; la variedad evaluada es el clon de Cacao tipo forastero CCN-51 con cinco años de desarrollo, con un marco de siembra de tres por tres metros en un arreglo a cuatro vientos y una densidad de 1,100 plantas/ha. La zona presenta un clima Tropical húmedo según clasificación de Holdribge, ubicada en las coordenadas geográficas este 658731 y norte 9811413 (PSDA), con temperatura media anual de $25.2^{\circ} \mathrm{C}$, una precipitación media anual de $1,887.75$ $\mathrm{mm}$ y humedad relativa de 78.9\%. El suelo de la zona es Franco Arcilloso de origen volcánico con clasificación Alfisol, estructura blocosa subangular, color café oscuro, profundidad media de 1.5 metros. 
Los tratamientos evaluados se obtuvieron de las combinaciones de los siguientes factores: Formas de aplicación: sobre superficie, bajo superficie. Dosis Fertilizante Nitrogenado: 50-100-150-200 kg/ha; Dosis Fertilizante Fosfatado: $35-70 \mathrm{~kg} / \mathrm{ha}$.

Tabla 1. Tratamientos

\begin{tabular}{|c|c|c|c|}
\hline & $\begin{array}{l}\text { Forma de } \\
\text { aplicación }\end{array}$ & $\begin{array}{l}\text { Fertilizante Nitrogenado } \\
\mathrm{kg} / \mathrm{ha}\end{array}$ & $\begin{array}{c}\text { Fertilizante Fosfatado } \\
\mathrm{kg} / \mathrm{ha}\end{array}$ \\
\hline $\mathrm{T} 1$ & \multirow{8}{*}{$\begin{array}{l}\text { Sobre } \\
\text { Superficie }\end{array}$} & 50 & 35 \\
\hline $\mathrm{T} 2$ & & 50 & 70 \\
\hline T3 & & 100 & 35 \\
\hline $\mathrm{T} 4$ & & 100 & 70 \\
\hline T5 & & 150 & 35 \\
\hline T6 & & 150 & 70 \\
\hline $\mathrm{T} 7$ & & 200 & 35 \\
\hline T8 & & 200 & 70 \\
\hline T9 & \multirow{8}{*}{$\begin{array}{l}\text { Bajo } \\
\text { Superficie }\end{array}$} & 50 & 35 \\
\hline T10 & & 50 & 70 \\
\hline T11 & & 100 & 35 \\
\hline T12 & & 100 & 70 \\
\hline $\mathrm{T} 13$ & & 150 & 35 \\
\hline T14 & & 150 & 70 \\
\hline $\mathrm{T} 15$ & & 200 & 35 \\
\hline T16 & & 200 & 70 \\
\hline T17 & Control & Sin aplicación & fertilizantes \\
\hline
\end{tabular}

Para el trabajo de investigación se utilizó el diseño experimental Bloques Completamente al Azar con arreglo factorial 2x4x2+1, en tres repeticiones. Los promedios de los resultados se compararon entre sí con la prueba de Tukey al 95\% de confianza, con la finalidad de conocer la diferencia estadística entre tratamientos. Las variables evaluadas fueron: diámetro de mazorca, longitud de mazorca, número de mazorcas por árbol, índice de semillas y rendimiento por hectárea de almendras, conteo de esporas, porcentaje de colonización de micorrizas, densidad visual del endófito, caracterización de especies micorrízicas.

Previo al establecimiento del ensayo se tomaron muestras de suelos, para proceder al análisis físico y químico; determinando así la cantidad de nutrientes existentes. Dentro del manejo del ensayo se realizó el control de malezas cada 30 días manualmente. No se efectuó control de insectos debido a la no presencia de los mismos en el cultivo durante el ensayo. Para controlar la enfermedad monilia (Moniliophthora roreri) que afecta las mazorcas de cacao, se ejecutó la aplicación de sulfato de cobre $(0,5$ 1/ha) dirigida a las mazorcas a los 40 y 80 días después de la instalación del ensayo. El primer ciclo de poda fitosanitaria se hizo al inicio del trabajo, el segundo ciclo de podas a los 90 días después, se empleó tijeras para poda, serruchos curvos y 
una formulación de pasta cúprica. Para satisfacer las necesidades hídricas del cultivo en la epoca seca el riego se lo aplico por surcos; en cada campaña de riego se efectuó la fertilización química en el ensayo. Las dosis de fertilización química se aplicaron según los tratamientos planteados, mensualmente mezclando cada producto y distribuyendo de forma manual dirigida a 0.5 metros del tronco en media-luna (66.3 g/planta/mes de mezcla). Los microelementos se aplicaron de manera foliar con los productos Metalosato Boro y Metalosato Zinc en dosis de 300cc/ha cada dos meses. La cosecha se realizó con barridos semanales por cada tratamiento de forma manual con tijeras y palancas cortas. Para abrir los frutos se utilizó un rabón, las semillas se fermentaron durante ocho días en sistema de montón rotatorio y secaron naturalmente sobre tendal.

El análisis poblacional e identificación de micorrizas, se realizó en los laboratorios de microbiología del CIPAL (ANCUPA, 2016), encontrando los géneros Glomus mosseae, Glomus fasciculatum, Gigaspora gigantea y Acaulospora mellea. Con esto se procedió al conteo poblacional inicial de esporas. Para el conteo de las esporas de micorrizas se utilizó el método de "tamizado en húmedo y decantación" de Gerdemann y Nicolson (1963) y la densidad visual del endófito fue evaluado con la metodología de Herrera (1993). En la identificación de especies se empleó la clasificación en base a morfología de las esporas proporcionadas por el INVAM (2016). El porcentaje de colonización de micorrizas, se estimó como el rendimiento de la micorriza en las plantas tratadas versus las plantas no tratadas, bajo un determinado nivel de infección, con la siguiente expresión:

$\mathrm{DM}=(\mathrm{M}-\mathrm{NM}) / \mathrm{NM} \mathrm{X} 100$

M: Rendimiento de la planta tratada

NM: Rendimiento de la planta no tratada

El número de mazorcas por árbol, se calculó en función del número de mazorcas provenientes de cinco arboles por tratamiento, en los cuales se contabilizó el total de frutos comerciales obtenidas durante el periodo del ensayo. El rendimiento por hectárea fue tomado en función del rendimiento de almendras secas por árbol y la población existente en el ensayo $(1,110$ plantas/ha).

\section{Resultados}

\section{Diámetro y longitud de mazorcas}

El diámetro de mazorcas reportó altas diferencias significativas para sistemas de aplicación y fertilizantes, no observándose en las interacciones entre ambos, siendo el coeficiente de variación fue $5.11 \%$ (Tabla 2). Aplicando la fertilización bajo superficie se presentó diferencias estadísticas 
altas, superior a las mazorcas provenientes del sistema sobre superficie (9.89 $\mathrm{cm})$, teniendo las plantas tratadas con $100 \mathrm{~kg} / \mathrm{ha} \mathrm{N}+35 \mathrm{~kg} / \mathrm{ha} \mathrm{P}(11.61 \mathrm{~cm})$ significancia mayor a la presentada en el testigo. El análisis de varianza en la longitud de mazorca, no determinó diferencias significativas en los datos de sistemas de aplicación, fertilizantes e interacciones (Tabla 2). Plantas tratadas con fertilización de $100 \mathrm{~kg} / \mathrm{ha} \mathrm{N}+70 \mathrm{~kg} / \mathrm{ha} \mathrm{P}(21.01 \mathrm{~cm})$ tuvieron mayor tamaño con relación a las plantas del testigo $(19.41 \mathrm{~cm})$.

Tabla 2. Valores promedios de diámetro y longitud de mazorcas.

\begin{tabular}{|c|c|c|c|}
\hline Sistema & $\begin{array}{l}\text { Fertilizante Aplicado } \\
\mathrm{N}+\mathrm{P} \mathrm{kg} / \mathrm{ha}\end{array}$ & $\begin{array}{l}\text { Diámetro } \\
\mathrm{cm}\end{array}$ & $\begin{array}{l}\text { Longitud } \\
\mathrm{cm}\end{array}$ \\
\hline Sobre superficie & & $9.89 \mathrm{~b}$ & $20.24 \mathrm{~ns}$ \\
\hline Bajo superficie & & $9.99 \mathrm{a}$ & 20.12 \\
\hline & $50+35$ & $9.49 \mathrm{~b}$ & $19.71 \mathrm{~ns}$ \\
\hline & $50+70$ & $10.27 \mathrm{ab}$ & 19.51 \\
\hline & $100+35$ & $11.61 \mathrm{a}$ & 19.95 \\
\hline & $100+70$ & $10.05 \mathrm{ab}$ & 21.01 \\
\hline & $150+35$ & $9.60 \mathrm{~b}$ & 20.34 \\
\hline & $150+70$ & $9.60 \mathrm{~b}$ & 20.57 \\
\hline & $200+35$ & $9.56 \mathrm{~b}$ & 19.56 \\
\hline & $200+70$ & $9.48 \mathrm{~b}$ & 19.76 \\
\hline & Testigo & $8.34 \mathrm{c}$ & 19.41 \\
\hline Sobre superficie & $50+35$ & $9.44^{\mathrm{ns}}$ & $20.28^{\mathrm{ns}}$ \\
\hline Sobre superficie & $50+70$ & 10.22 & 20.13 \\
\hline Sobre superficie & $100+35$ & 10.56 & 20.32 \\
\hline Sobre superficie & $100+70$ & 10.00 & 20.07 \\
\hline Sobre superficie & $150+35$ & 9.56 & 19.76 \\
\hline Sobre superficie & $150+70$ & 9.56 & 19.67 \\
\hline Sobre superficie & $200+35$ & 9.54 & 19.86 \\
\hline Sobre superficie & $200+70$ & 10.32 & 19.87 \\
\hline Bajo superficie & $50+35$ & 10.66 & 19.55 \\
\hline Bajo superficie & $50+70$ & 10.10 & 19.80 \\
\hline Bajo superficie & $100+35$ & 9.65 & 19.97 \\
\hline Bajo superficie & $100+70$ & 9.65 & 20.36 \\
\hline Bajo superficie & $150+35$ & 10.56 & 19.67 \\
\hline Bajo superficie & $150+70$ & 10.68 & 19.56 \\
\hline Bajo superficie & $200+35$ & 9.67 & 19.78 \\
\hline Bajo superficie & $200+70$ & 9.77 & 19.45 \\
\hline Testigo & & 8.34 & 19.41 \\
\hline \multicolumn{2}{|l|}{ Promedio General } & 9.94 & 19.78 \\
\hline & Factor A (Sistema de Sombreo) & $* *$ & Ns \\
\hline & Factor B (Dosis Micorrizas) & $* *$ & Ns \\
\hline & Interacción $(\mathrm{A} \times \mathrm{B})$ & Ns & Ns \\
\hline \multicolumn{2}{|c|}{ Coeficiente de variación $(\%)$} & 5.11 & 4.82 \\
\hline
\end{tabular}

\section{Número de mazorcas por árbol e índice de semilla}

El análisis de varianza encontró altas diferencias significativas en estas variables (Tabla 3). El sistema bajo superficie dio mayor cantidad de mazorcas e índice de semillas (62.87 mazorcas/árbol y $121.17 \mathrm{~g})$, superior 
estadísticamente a la aplicación sobre superficie (58.54 mazorcas/árbol). Aplicando fertilización química $100 \mathrm{~kg} / \mathrm{ha} \mathrm{N}+70 \mathrm{~kg} / \mathrm{ha} \mathrm{P}$, se logra mayor número de mazorcas (68.14 mazorcas/árbol) y semillas más pesadas (121.17 $\mathrm{g})$, superior a las otras dosis de fertilizantes. Las interacciones mostraron que aplicar el fertilizante bajo superficie en dosis de $100 \mathrm{~kg} / \mathrm{ha} \mathrm{N}+70 \mathrm{~kg} / \mathrm{ha} \mathrm{P}$, presenta mayor número de mazorcas (74.61 mazorcas) e índice de semilla (128.8 g). Todos los tratamiento fueron superiores al testigo (Tukey <0,05), hecho que hace resaltar lo manifestado por Casas (2011), quien habla sobre el rol de fertilización balanceada en los cultivos y la importancia de la conservación del recurso suelos, a través del manejo agronómico de las aportaciones.

Tabla 3. Valores promedios de número de mazorcas por árbol e índice de semilla.

\begin{tabular}{|c|c|c|c|}
\hline Sistema & $\begin{array}{l}\text { Fertilizante Aplicado } \\
\mathrm{N}+\mathrm{P} \mathrm{kg} / \mathrm{ha}\end{array}$ & $\begin{array}{l}\text { Mazorcas } \\
\text { por árbol }\end{array}$ & $\begin{array}{l}\text { Índice de } \\
\text { semilla } g\end{array}$ \\
\hline Sobre superficie & & $58.54 \mathrm{~b}$ & $118.74 \mathrm{~b}$ \\
\hline \multirow[t]{10}{*}{ Bajo superficie } & & $62.87 \mathrm{a}$ & $121.17 \mathrm{a}$ \\
\hline & $50+35$ & $62.16 \mathrm{~b}$ & $119.53 \mathrm{~b}$ \\
\hline & $50+70$ & $58.65 \mathrm{~b}$ & $116.75 \mathrm{c}$ \\
\hline & $100+35$ & $63.45 \mathrm{~b}$ & $117.48 \mathrm{c}$ \\
\hline & $100+70$ & $68.14 \mathrm{a}$ & $125.07 \mathrm{a}$ \\
\hline & $150+35$ & $62.98 \mathrm{~b}$ & $120.42 \mathrm{~b}$ \\
\hline & $150+70$ & $58.34 \mathrm{~b}$ & $117.45 \mathrm{c}$ \\
\hline & $200+35$ & $59.56 \mathrm{~b}$ & $119.56 \mathrm{~b}$ \\
\hline & $200+70$ & $59.48 \mathrm{~b}$ & $119.76 \mathrm{~b}$ \\
\hline & Testigo & $48.83 \mathrm{c}$ & $113.41 \mathrm{~d}$ \\
\hline Sobre superficie & $50+35$ & $60.79 \mathrm{~b}$ & $120.73 \mathrm{~cd}$ \\
\hline Sobre superficie & $50+70$ & $57.36 \mathrm{bc}$ & $117.93 \mathrm{de}$ \\
\hline Sobre superficie & $100+35$ & $62.06 \mathrm{~b}$ & $118.67 \mathrm{de}$ \\
\hline Sobre superficie & $100+70$ & $61.59 \mathrm{~b}$ & $118.67 \mathrm{de}$ \\
\hline Sobre superficie & $150+35$ & $61.66 \mathrm{~b}$ & $121.67 \mathrm{bc}$ \\
\hline Sobre superficie & $150+70$ & $57.75 \mathrm{bc}$ & $121.33 \mathrm{bc}$ \\
\hline Sobre superficie & $200+35$ & $63.52 \mathrm{~b}$ & $118.32 \mathrm{de}$ \\
\hline Sobre superficie & $200+70$ & $59.95 \mathrm{~b}$ & $115.57 \mathrm{e}$ \\
\hline Bajo superficie & $50+35$ & $64.85 \mathrm{ab}$ & $116.29 \mathrm{e}$ \\
\hline Bajo superficie & $50+70$ & $64.36 \mathrm{~b}$ & $116.29 \mathrm{e}$ \\
\hline Bajo superficie & $100+35$ & $61.45 \mathrm{~b}$ & $122.17 \mathrm{bc}$ \\
\hline Bajo superficie & $100+70$ & $74.61 \mathrm{a}$ & $128.8 \mathrm{a}$ \\
\hline Bajo superficie & $150+35$ & $62.45 \mathrm{~b}$ & $121.09 \mathrm{bc}$ \\
\hline Bajo superficie & $150+70$ & $60.68 \mathrm{~b}$ & $119.56 \mathrm{~d}$ \\
\hline Bajo superficie & $200+35$ & $59.67 \mathrm{~b}$ & $119.78 \mathrm{~d}$ \\
\hline Bajo superficie & $200+70$ & $59.77 \mathrm{~b}$ & $119.45 \mathrm{~d}$ \\
\hline Testigo & & $47.75 \mathrm{c}$ & $113.41 \mathrm{f}$ \\
\hline \multirow[t]{4}{*}{ Promedio General } & & 60.70 & 119.96 \\
\hline & Factor A (Sistema de Sombreo) & $* *$ & $* *$ \\
\hline & Factor B (Dosis Micorrizas) & $* *$ & $* *$ \\
\hline & Interacción $(\mathrm{A} \times \mathrm{B})$ & $* *$ & $* *$ \\
\hline \multicolumn{2}{|c|}{ Coeficiente de variación $(\%)$} & 5.47 & 0.88 \\
\hline
\end{tabular}




\section{Rendimiento por hectárea de almendras}

Los promedios del rendimiento de almendras por hectárea, determinaron altas diferencias significativas en los factores evaluados (Tabla 4). El sistema de aplicación bajo superficie estadísticamente logró el mayor rendimiento de grano $(1,154.93 \mathrm{~kg} / \mathrm{ha})$, observándose el tratamiento $100 \mathrm{~kg} / \mathrm{ha}$ $\mathrm{N}+70 \mathrm{~kg} / \mathrm{ha} \mathrm{P}(1,638.09 \mathrm{~kg} /)$ con más rendimiento comparados con los otros programas de fertilización. Cuando se colocó bajo superficie $100 \mathrm{~kg} / \mathrm{ha} \mathrm{N}+$ $70 \mathrm{~kg} / \mathrm{ha} \mathrm{P}$, se alcanzó incrementos de rendimiento $(1,572.40 \mathrm{~kg} / \mathrm{ha})$, mayor estadísticamente a los demás tratamientos.

Tabla 4. Rendimiento por hectárea de almendras.

\begin{tabular}{|c|c|c|}
\hline Sistema & $\begin{array}{l}\text { Fertilizante Aplicado } \\
\mathrm{N}+\mathrm{P}(\mathrm{kg} / \mathrm{ha})\end{array}$ & $\mathrm{Kg} / \mathrm{ha}$ \\
\hline Sobre superficie & & $1,124.89 \mathrm{~b}$ \\
\hline \multirow[t]{10}{*}{ Bajo superficie } & & $1,154.93 \mathrm{a}$ \\
\hline & $50+35$ & $1,107.21 \mathrm{e}$ \\
\hline & $50+70$ & $1,245.47 \mathrm{~b}$ \\
\hline & $100+35$ & $1,189.96 \mathrm{c}$ \\
\hline & $100+70$ & $1,638.09 \mathrm{a}$ \\
\hline & $150+35$ & $1,163.47 \mathrm{~d}$ \\
\hline & $150+70$ & $1,195.28 \mathrm{c}$ \\
\hline & $200+35$ & $999.56 \mathrm{c}$ \\
\hline & $200+70$ & $979.76 \mathrm{c}$ \\
\hline & Testigo & $795.28 \mathrm{f}$ \\
\hline Sobre superficie & $50+35$ & $1,135.60 \mathrm{de}$ \\
\hline Sobre superficie & $50+70$ & $1,277.40 \mathrm{~b}$ \\
\hline Sobre superficie & $100+35$ & $1,176.8 \mathrm{c}$ \\
\hline Sobre superficie & $100+70$ & $1,220.47 \mathrm{c}$ \\
\hline Sobre superficie & $150+35$ & $1,108.07$ ef \\
\hline Sobre superficie & $150+70$ & $815.67 \mathrm{~g}$ \\
\hline Sobre superficie & $200+35$ & $1,078.82 \mathrm{f}$ \\
\hline Sobre superficie & $200+70$ & $1,213.53 \mathrm{c}$ \\
\hline Bajo superficie & $50+35$ & $1,303.78 \mathrm{~b}$ \\
\hline Bajo superficie & $50+70$ & $1,159.44 \mathrm{~d}$ \\
\hline Bajo superficie & $100+35$ & $1,218.87 \mathrm{c}$ \\
\hline Bajo superficie & $100+70$ & $1,572.40 \mathrm{a}$ \\
\hline Bajo superficie & $150+35$ & $1,211.09 \mathrm{bc}$ \\
\hline Bajo superficie & $150+70$ & $1,129.56 \mathrm{~d}$ \\
\hline Bajo superficie & $200+35$ & $1,189.78 \mathrm{~d}$ \\
\hline Bajo superficie & $200+70$ & $1,119.45 \mathrm{~d}$ \\
\hline Testigo & & $795.28 \mathrm{~h}$ \\
\hline \multirow[t]{4}{*}{ Promedio General } & & $1,130.91$ \\
\hline & Factor A (Sistema de Sombreo) & $* *$ \\
\hline & Factor B (Dosis Micorrizas) & $* *$ \\
\hline & Interacción $(A \times B)$ & $* *$ \\
\hline \multicolumn{2}{|c|}{ Coeficiente de variación $(\%)$} & 1.18 \\
\hline
\end{tabular}




\section{Conteo de esporas}

El conteo de esporas, dio alta significancia estadística en las evaluaciones realizadas al inicio y final de la investigación (tabla 5). La evaluación inicial encontró mayor cantidad de esporas en el sistema de aplicación bajo superficie con 106.44 espora/gss. Así mismo los lotes donde se colocó $100 \mathrm{~kg} / \mathrm{ha} \mathrm{N}+70 \mathrm{~kg} / \mathrm{ha} \mathrm{P}$ presentaron mayor cantidad de esporas 106.93 esporas/gss, superiores estadísticamente superior a los demás tratamientos. De la misma manera la interacción entre la aplicación bajo superficie y la fertilización con $100 \mathrm{~kg} / \mathrm{ha} \mathrm{N}+70 \mathrm{~kg} / \mathrm{ha} \mathrm{P}$ (107.98 esporas/gss) fue igual estadísticamente todos los tratamientos, excepto a la aplicación bajo superficie fertilizado con $50 \mathrm{~kg} / \mathrm{ha} \mathrm{N}+35 \mathrm{~kg} / \mathrm{ha} \mathrm{P}$ (103.27 esporas/gss). Al final del ensayo, el conteo de esporas reportó mayor cantidad, cuando se aplicó fertilizante bajo superficie del suelo (150.34 espora/gss). Además aplicando $100 \mathrm{~kg} / \mathrm{ha} \mathrm{N}+70 \mathrm{~kg} / \mathrm{ha} \mathrm{P}$, se tiene más esporas (158.57); viéndose también el mismo efecto en la interacción de la aplicación bajo superficie con el programa de aplicación de $100 \mathrm{~kg} / \mathrm{ha} \mathrm{N}+70 \mathrm{~kg} / \mathrm{ha} \mathrm{P}$ (179.18 esporas/gss), que fue superior a todos los tratamientos. Los datos demuestran que las micorrizas y en especial el género Glomus, presentan un alto índice de número de esporas, lo que demuestra la facilidad que tienen los representantes de este género para colonizar raíces de cacao. Esto concuerda con lo descrito por Prieto et al. (2012), quien además sostiene que se conoce muy poco sobre estos microorganismos benéficos

Tabla 5. Conteo de esporas.

\begin{tabular}{lccc}
\hline \multirow{2}{*}{ Sistema } & $\begin{array}{c}\text { Fertilizante Aplicado } \\
\mathrm{N}+\mathrm{P}(\mathrm{kg} / \mathrm{ha})\end{array}$ & $\begin{array}{c}\mathbf{0} \\
\text { d.d.i }\end{array}$ & $\begin{array}{c}\mathbf{1 2 0} \\
\mathbf{d . d . i}\end{array}$ \\
\hline & & \multicolumn{2}{c}{ esporas/gss } \\
Sobre superficie & & $104.36 \mathrm{~b}$ & $119.87 \mathrm{~b}$ \\
Bajo superficie & & $106.44 \mathrm{a}$ & $150.34 \mathrm{a}$ \\
\hline $50+35$ & & $103.57 \mathrm{~b}$ & $129.69 \mathrm{~d}$ \\
$50+70$ & & $103.45 \mathrm{~b}$ & $124.56 \mathrm{~d}$ \\
$100+35$ & & $103.11 \mathrm{~b}$ & $150.25 \mathrm{~b}$ \\
$100+70$ & & $106.93 \mathrm{a}$ & $158.57 \mathrm{a}$ \\
$150+35$ & & $104.30 \mathrm{~b}$ & $150.25 \mathrm{~b}$ \\
$150+70$ & & $104.04 \mathrm{~b}$ & $144.95 \mathrm{c}$ \\
$200+35$ & & $103.57 \mathrm{~b}$ & $114.78 \mathrm{e}$ \\
$200+70$ & & $104.38 \mathrm{~b}$ & $112.40 \mathrm{e}$ \\
Testigo & & $104.08 \mathrm{~b}$ & $141.61 \mathrm{c}$ \\
\hline Sobre superficie & $50+35$ & $105.87 \mathrm{abc}$ & $148.21 \mathrm{~d}$ \\
Sobre superficie & $50+70$ & $105.40 \mathrm{abc}$ & $137.97 \mathrm{e}$ \\
Sobre superficie & $100+35$ & $104,07 \mathrm{abc}$ & $171.71 \mathrm{~b}$ \\
Sobre superficie & $100+70$ & $104.15 \mathrm{abc}$ & $161.65 \mathrm{~b}$ \\
Sobre superficie & $150+35$ & $104.60 \mathrm{abc}$ & $116.82 \mathrm{~g}$ \\
Sobre superficie & $150+70$ & $101.33 \mathrm{abc}$ & $112.48 \mathrm{hi}$ \\
Sobre superficie & $200+35$ & $104.08 \mathrm{abc}$ & $102.74 \mathrm{j}$ \\
Sobre superficie & $200+70$ & $104.15 \mathrm{abc}$ & $82.33 \mathrm{k}$
\end{tabular}




\begin{tabular}{lccc} 
Sobre superficie & $50+35$ & $103.53 \mathrm{bc}$ & $165.65 \mathrm{~b}$ \\
Bajo superficie & $50+35$ & $103.27 \mathrm{c}$ & $120.82 \mathrm{~g}$ \\
Bajo superficie & $50+70$ & $104.00 \mathrm{abc}$ & $116.48 \mathrm{hi}$ \\
Bajo superficie & $100+35$ & $107.51 \mathrm{ab}$ & $111.16 \mathrm{ij}$ \\
Bajo superficie & $100+70$ & $107.98 \mathrm{a}$ & $179.18 \mathrm{a}$ \\
Bajo superficie & $150+35$ & $106.15 \mathrm{abc}$ & $128.78 \mathrm{f}$ \\
Bajo superficie & $150+70$ & $105.60 \mathrm{abc}$ & $124.24 \mathrm{fg}$ \\
Bajo superficie & $200+35$ & $105.33 \mathrm{abc}$ & $108.74 \mathrm{j}$ \\
Bajo superficie & $200+70$ & $104.08 \mathrm{abc}$ & $141.61 \mathrm{~d}$ \\
Testigo & & \\
\hline $\begin{array}{l}\text { Promedio General } \\
\text { Factor A (Sistema de Sombreo) }\end{array}$ & 101.40 & 131.11 \\
Factor B (Dosis Micorrizas) & $* *$ & $* *$ \\
Interacción (A x B) & $* *$ & $* *$ \\
Coeficiente de variación (\%) & $* *$ & 2.41 \\
\hline
\end{tabular}

\section{Porcentaje de colonización de micorrizas}

El mayor porcentaje de colonización del hongo se dio en el sistema de aplicación bajo superficie con $44.84 \%$ (tabla 6). El tratamiento aplicado con $100 \mathrm{~kg} / \mathrm{ha} \mathrm{N}+70 \mathrm{~kg} / \mathrm{ha} \mathrm{P}(68.13 \%)$ fue estadísticamente superior a los otros tratamientos. Las interacciones mostraron significancia en sistema bajo sombra tratado con $100 \mathrm{~kg} / \mathrm{ha} \mathrm{N}+70 \mathrm{~kg} / \mathrm{ha} \mathrm{P}(67.62 \%)$, que fue estadísticamente superior. Esto se relaciona con los resultados obtenidos por Brundrett et al. (2006), que según la presencia del hongo en las raíces, se modifica su morfología, promoviendo la ramificación y aumentando con esto su superficie de absorción.

Tabla 6. Porcentaje de colonización de micorrizas.

\begin{tabular}{lcc}
\hline \multicolumn{1}{c}{ Sistema } & $\begin{array}{c}\text { Fertilizante Aplicado } \\
\mathrm{N}+\mathrm{P}(\mathrm{kg} / \mathrm{ha})\end{array}$ & $\%$ \\
\hline Sobre superficie & & $41.67^{\mathrm{ns}}$ \\
Bajo superficie & $50+35$ & 44.84 \\
\hline & $50+70$ & $38.83 \mathrm{c}$ \\
& $100+35$ & $56.51 \mathrm{ab}$ \\
& $100+70$ & $49.53 \mathrm{bc}$ \\
& $150+35$ & $68.13 \mathrm{a}$ \\
& $150+70$ & $46.53 \mathrm{bc}$ \\
& $200+35$ & $40.00 \mathrm{bc}$ \\
& $200+70$ & $9.18 \mathrm{~d}$ \\
& $\mathrm{Testigo}$ & $5.01 \mathrm{~d}$ \\
& $50+35$ & $47.15 \mathrm{bc}$ \\
\hline Sobre superficie & $50+70$ & $39.09 \mathrm{bc}$ \\
Sobre superficie & $100+35$ & $36.84 \mathrm{bc}$ \\
Sobre superficie & $100+70$ & $38.65 \mathrm{bc}$ \\
Sobre superficie & $150+35$ & $39.54 \mathrm{bc}$ \\
Sobre superficie & $150+70$ & $5.91 \mathrm{~d}$ \\
Sobre superficie & $200+35$ & $4.21 \mathrm{~d}$ \\
Sobre superficie & $200+70$ & $38.57 \mathrm{bc}$ \\
Sobre superficie & & $36.19 \mathrm{bc}$
\end{tabular}




\begin{tabular}{lcc} 
Bajo superficie & $50+35$ & $49.62 \mathrm{bc}$ \\
Bajo superficie & $50+70$ & $49.52 \mathrm{bc}$ \\
Bajo superficie & $100+35$ & $57.14 \mathrm{ab}$ \\
Bajo superficie & $100+70$ & $67.62 \mathrm{a}$ \\
Bajo superficie & $150+35$ & $47.35 \mathrm{bc}$ \\
Bajo superficie & $150+70$ & $36.19 \mathrm{bc}$ \\
Bajo superficie & $200+35$ & $48.32 \mathrm{bc}$ \\
Bajo superficie & $200+70$ & $47.22 \mathrm{bc}$ \\
Testigo & & $52.14 \mathrm{ab}$ \\
\hline Promedio General & & 48.16 \\
& Factor A (Sistema de Sombreo) & $* *$ \\
& Factor B (Dosis Micorrizas) & $* *$ \\
Coeficiente de variación (\%) & Interacción (A x B) & $* *$ \\
& & 18.10 \\
\hline
\end{tabular}

\section{Densidad del endófito}

La variable densidad del endófito tuvo diferencias altamente significativas, según el análisis de varianza y la prueba de tukey al $95 \%$ de confianza, para los sistemas de aplicación, dosis de fertilizantes e interacciones (Figura 1). La aplicación bajo superficie con 2.59 (Herrera, 1993), fue estadísticamente superior a colocar el fertilizante sobre la superficie del suelo. Aplicando $100 \mathrm{~kg} / \mathrm{ha} \mathrm{N}+70 \mathrm{~kg} / \mathrm{ha} \mathrm{P}$ se logró más densidad del hongo, con relación al resto de programas de fertilización, siendo estadísticamente superior. Las interacciones demostraron mayor desarrollo del endófito cuando se aplicó el fertilizante bajo la superficie en dosis de 100 $\mathrm{kg} / \mathrm{ha} \mathrm{N}+70 \mathrm{~kg} / \mathrm{ha} \mathrm{P}$ (3.27); siendo superior estadísticamente a las demás relaciones.

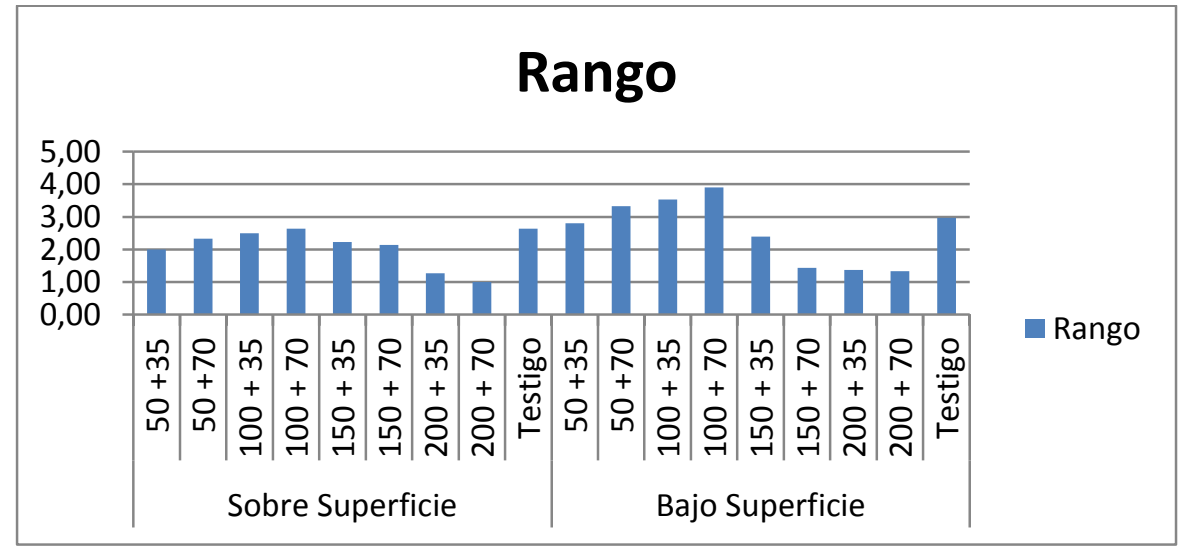

Figura 1. Densidad de endófito con la aplicación de nitrógeno y fósforo en el cultivo de cacao.

\section{Correlación dosis de fertilizante vs. Densidad del endófito}

Las correlaciones mostraron una relación negativa entre el nutriente aplicado y el número de esporas de micorrizas presentes en el suelo. Es decir 
la disminución de esporas fue significativa a medida que las dosis de fertilizantes nitrogenados y fosforados aumentaron. La reducción de esporas estuvo entre el 30,66 al 54,05\% con las dosis mayores a $200 \mathrm{~kg} / \mathrm{ha}$ de $\mathrm{N}$ y 70 $\mathrm{kg} /$ ha de $\mathrm{P}$ (Figura 2). Esto concuerda con los resultados encontrados por Ballesteros et al. (2004), quienes obtuvieron más infectividad de Glomus en dosis de $50 \mathrm{~kg}$ de $\mathrm{P} / \mathrm{ha}$, presentando un $31 \%$ de infección, comparado con los tratamientos sin inoculación de micorrizas y sin aplicación de fósforo.

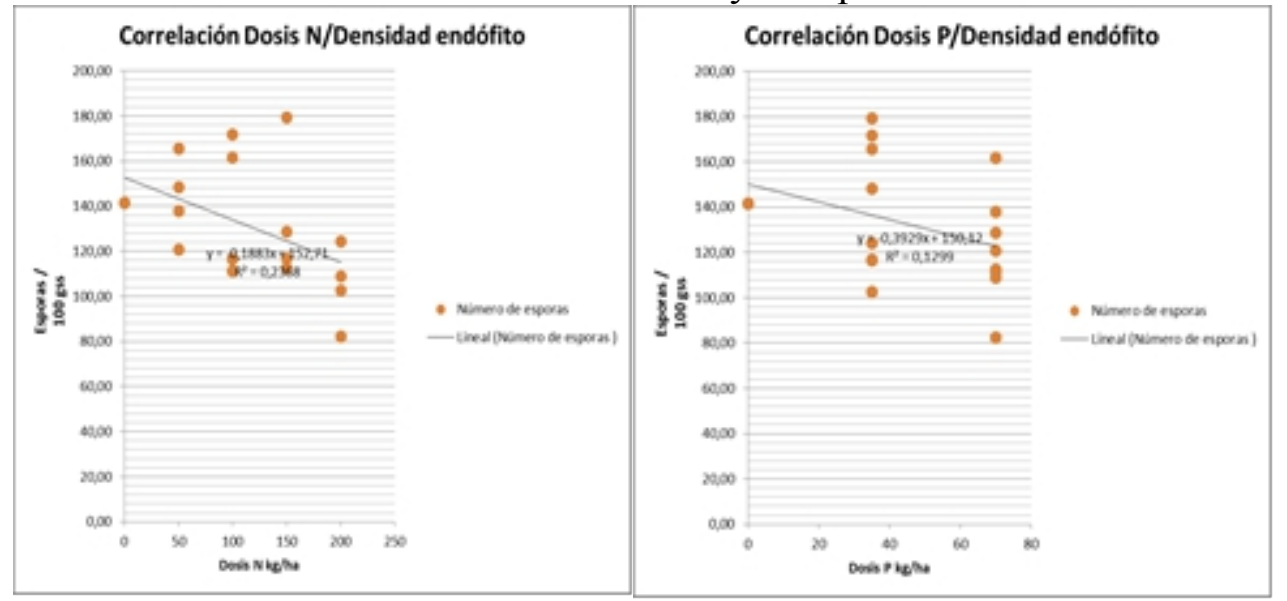

Figura 2. Correlación entre dosis de fertilizantes y número de esporas en el cultivo de cacao.

\section{Discusión}

Los resultados de la presente investigación indican que la aplicación de fertilizantes nitrogenados y fosforados sobre el suelo o enterrados, tienen influencia sobre las poblaciones de micorrizas. Dosis altas generan una influencia marcada en las poblaciones de hongos asociados a cacao, esencialmente sobre la población de esporas y porcentaje de colonización, mostrando en dosis medias a bajas poca afectación y con las altas disminución de estas variables. Esto concuerda por lo manifestado Sieverding (2007), quien menciona que se requiere de su manejo especial para las poblaciones del hongo, debido principalmente a que la distribución de los hongos micorrízicos en el suelo no es homogénea y, hay sitios donde la concentración de estos hongos es muy baja para una producción de biomasa de plantas óptima.

Los resultados determinan una respuesta agronómica positiva, cuando se aplica $100 \mathrm{~kg} / \mathrm{ha}$ de $\mathrm{N}$ y $70 \mathrm{~kg} / \mathrm{ha} \mathrm{P}$, además de enterrar el fertilizante. Esto logra una mejor interrelación entre el nutriente y el organismo que lo transforma en el suelo, lo que permite la maximización de especies micorrízicas mejor adaptadas a la particularidades del entorno, como lo corrobora Pérez (2000), quien dice que con la utilización de las micorrizas como biofertilizantes no se suprime la aplicación de fertilizantes, sino que la fertilización se hace más eficiente y puede disminuirse la dosis. Así mismo 
López et al. (2007), encontraron que el contenido de fósforo disponible afecta el número de esporas de hongos, ocurriendo la disminución del número de esporas al incrementarse las dosis de $\mathrm{P}$, esto podría crear dependencia de insumos externos en detrimento de los procesos biológicos naturales como la simbiosis micorrízica.

El incremento de rendimiento de frutos con la aplicación de fertilizantes maximiza la eficiencia de los nutrientes aportados, si no se colocan fertilizantes el mismo no puede por sí solo garantizar la nutrición a la planta. Esto ratifica lo manifestado por Bernal y Morales (2006), quienes expresan que los hongos extienden el micelio en el suelo para extraer agua y elementos esenciales para compartirlos con las plantas. Las investigaciones muestran que las micorrizas incrementan la tolerancia de las plantas a la sequía, compactación, altas temperaturas del suelo, metales pesados, salinidad, toxinas orgánicas e inorgánicas y extremos de $\mathrm{pH}$ del suelo, con lo que prolongan la vida, viabilidad y productividad del sistema radical de la planta.

La producción de almendras se dio en el sistema de aplicación bajo superficie cuando se aplicó $100 \mathrm{~kg} /$ ha de $\mathrm{N}+70 \mathrm{~kg} / \mathrm{ha} \mathrm{P}$, con lo que se logra aumento no solo el rendimiento, sino también la concentración de esporas en el suelo. Lo que concuerda con Pérez y Vertel (2009), quienes dicen que como recurso biológico las micorrizas generan beneficios al mejorar las condiciones físico-químicas y biológicas del suelo. Los beneficios desde el punto de visita bilógico, se derivan de su interacción con los diversos grupos de macro y microorganismos de la rizósfera, tales como aquellos implicados en el ciclaje de nutrientes.

\section{Conclusiones}

$>$ En los suelos del cultivo evaluado, se identificaron los géneros Glomus mosseae, Glomus fasciculatum, Gigaspora gigantea y Acaulospora mellea.

$>$ El sistema de aplicación bajo superficie y la aplicación de fertilización química nitrogenada y fosforada, influyó sobre las poblaciones del hongo.

$>$ El uso de fertilizantes en dosis de $100 \mathrm{~kg} / \mathrm{ha} \mathrm{N}+70 \mathrm{~kg} / \mathrm{ha} \mathrm{P}$, aumentó el número de mazorcas.

$>$ Se producen aumentos en el peso almendras, aplicando fertilizantes nitrogenados y fosforados en de 100 y $70 \mathrm{~kg} / \mathrm{ha}$, respectivamente.

$>$ Hubo incrementos del 68\% más sobre el testigo absoluto, con la aplicación de $100 \mathrm{~kg} / \mathrm{ha} \mathrm{N}+70 \mathrm{~kg} / \mathrm{ha} \mathrm{P}$ en cacao. 
$>$ Aumentó el número de esporas por gramo de suelo, cuando se fertilizó el cultivo con $100 \mathrm{~kg} / \mathrm{ha} \mathrm{N}+70 \mathrm{~kg} / \mathrm{ha} \mathrm{P}$, encontrándose incrementos del $41 \%$ en relación al testigo sin aplicación.

$>$ Existió mayor colonización de micelio micorrízico en las raíces, cuando se aplicó fertilizantes bajo superficie y se aplicó $100 \mathrm{~kg} / \mathrm{ha} \mathrm{N}$ $+70 \mathrm{~kg} / \mathrm{ha} \mathrm{P}$; siendo la densidad del endófito mayor con la misma dosis y sistema de aplicación.

\section{References:}

1. Asociación de cultivadores de palma aceitera-ANCUPA. (2016). Informe técnico anual-CIPAL. Editado por Ancupa. Quito-Ecuador. 60 p. Recuperado de http:www.ancupa.com/informeanuales/tecnico.

2. ANECACAO. (2009). Manual del cultivo de cacao para pequeños productores. Editado por CORPEI. Vol. 1. Guayaquil-Ecuador. 90 p.

3. Bastidas, P., Peña, R., Reyes, C. (2005). Metodología de selección para mejoramiento genético acelerado de la palma de aceite. Prueba de campo. Revista fototécnica Colombiana 5, $\mathrm{n}^{\mathrm{o}} 1$ : 46-52.

4. Ballesteros, W., Unigarro, A., Cadena, C., Cadena, J. (2004). Evaluación de hongos formadores de micorrizas vesiculo arbusculares (MVA) en la etapa de almacigo de cacao (Theobroma cacao l.), en Tumaco, Nariño. Revista de Ciencias Agrícolas. Volumen XXI. Número I - II: 2004.

5. Bernal, G., Morales, R. 2006. Micorrizas. Importancia, producción e investigación en el Ecuador. Revista ANCUPA. 2006: 12-15.

6. Blanco, F. Salas, E. (2007). Micorrizas en la agricultura: Contexto mundial e investigación realizada en Costa Rica. Agronomía Costarricense 21. $\mathrm{n}^{\circ} 1$ : 55-67.

7. Brundrett, M., Bougher, N., Dell, B., Grove, T., Malajczuk, N. (2006). Working with mycorrhizas in forestry and agriculture. ACIAR Monograph, 32 (2006): 374.

8. Casas, F. (2011). Producción Agropecuaria en Latinoamérica. Editado por CIID. Producción Agropecuaria en el Paraguay. Lima: IICA, 2011. 107.

9. Gerdemann, J., Nicholson, T. (1963). Spores of Mycorrhizal endogene specie extracted from soil by wet sieving and decanting. British Mycological Society 46:235-244.

10. Herrera-Peraza, R. (1993). General methodology to analyze rootlets, raw humus and VA mycorrhizal (VAM) components. Cuba. p. 1-8.

11. Instituto Nacional de Investigaciones Agropecuarias- INIAP. (2010). Clones de cacao nacional para producción en el Ecuador. Boletín Divulgativo 348. Quevedo-Ecuador. 16 p. 
12. Instituto Nacional de Investigaciones Agropecuarias-INIAP. (2009). Manual de cultivo de cacao para la Amazonía ecuatoriana. Manual Vol. 4. Quevedo-Ecuador. 58 p.

13. International Culture collection of arbuscular \& vesicular arbuscular mycorrhizal. 2016. Fungi. Recuperado de http://www.invam.org/mycorrhizal

14. López, M., López de Rojas, I., España, M., Izquierdo, A., Herrera, L. (2007). Efecto de la fertilización inorgánica sobre la disponibilidad de nutrimentos en el suelo, nivel nutricional de la planta y hongos micorrízicos arbusculares en plantaciones de Theobroma cacao. Agronomía Trop. 57(1): 31-43.

15. Modjo, H., Hendrix, J., Jhonson, W., Rossi, G. 2006. The mycorrhizal fungus Glomus macrocarpum as a cause of tobacco stunt disease. Phytophatology. Recupetrado de http//search-pdfbooks.com/mycorrhizal fungus.

16. Pérez, A. Vertel, M. (2009). Evaluación de la colonización de micorrizas arbusculares en pasto Bothriochloa pestusa (L.). Revista MVZ-Córdova 15, no 3:(Sept-Dic 2009): 46.

17. Pérez, M. E. (2000). Tecnología para la eliminación del bromuro de metilo. Semillero de tabaco con substrato orgánico y uso de medios biológicos. Instituto de investigaciones de Sanidad Vegetal. MINAGRI, p. 16-30.

18. Prieto, O., Belezaca, C., Mora, W., Garcés, F. Sabando, F., Cedeño, P. (2012). Identificación de hongos micorrízicos arbusculares en sistemas agroforestales con cacao en el trópico húmedo ecuatoriano. Agronomía Mesoamericana 23(2):233-239. 2012. ISSN: 1021-7444

19. Sieverding, E. 2007. Vesicular-arbuscular mycorrhizal management in tropiacal agrosystems. Technical Cooperation. Federal Republic of Germany. P. 371. Recuperado de http//search-pdf-books.com/ mycorrhizal

20. Sylvia, D., Williams, E. (2001). Vesicular-arbuscular mycocchizae and enviromental stress. Vol. 1, cap. 3 de Mycorrhizae in sustainable agriculture, de GJ Bethlenfalvay y RG Linderman, 242. Wisconsi: ASA-CSSA-SSSA, 2001. 